\title{
Multiple Introductions of Multidrug-Resistant Tuberculosis into Households, Lima, Peru
}

\author{
Ted Cohen, Megan Murray, Ibrahim Abubakar, Zibiao Zhang, Alexander Sloutsky, Fernando Arteaga, \\ Katiuska Chalco, Molly F. Franke, and Mercedes C. Becerra
}

Two cases of multidrug-resistant tuberculosis (MDR TB) in a household are assumed to reflect within-household transmission. However, in high-incidence areas of MDR $T B$, secondary cases may arise through exposure to MDR TB in the community. To estimate the frequency of multiple introductions of MDR TB into households, we used spoligotyping and 24-loci mycobacterial interspersed repetitive unit-variable number tandem repeats to classify isolates from 101 households in Lima, Peru, in which >1 MDR TB patient received treatment during 1996-2004. We found different MDR TB strains in $\geq 10 \%$ of households. Alternate approaches for classifying matching strains produced estimates of multiple introductions in $\leq 38 \%$ of households. At least 4\% of MDR TB patients were reinfected by a second strain of MDR Mycobacterium tuberculosis. These findings suggest that community exposure to MDR TB in Lima occurs frequently. Rapid drug sensitivity testing of strains from household contacts of known MDR TB patients is needed to identify optimal treatment regimens.

The discovery and use of discriminating genetic 1 markers such as IS6110 restriction fragment length polymorphisms (RFLPs), spacer oligonucleotides (spoligotyping), and mycobacterial interspersed repetitive unit-variable number tandem repeats (MIRU-VNTRs)

Author affiliations: Brigham and Women's Hospital, Boston, Massachusetts, USA (T. Cohen, M. Murray, Z. Zhang, M.C. Becerra); Harvard School of Public Health, Boston (T. Cohen, M. Murray); University of East Anglia, Norwich, UK (I. Abubakar); Health Protection Agency Centre for Infections, London, UK (I. Abubakar); University of Massachusetts Medical School, Boston (A. Sloutsky); Partners In Health, Boston and Lima, Peru (F. Arteaga, K. Chalco, M.F. Franke, M.C. Becerra); and Harvard Medical School, Boston (M.F. Franke, M.C. Becerra)

DOI: $10.3201 /$ eid1706.101471
(1) have improved our understanding of the transmission dynamics of tuberculosis (TB) $(2,3)$. Genotyping studies, in which strains with matching sets of markers are considered potential members of a single transmission chain, have demonstrated that recent transmission plays a major role, even in low-incidence settings $(4,5)$; that persons with recurrent episodes of TB may be having reinfection rather than relapse (6-8); that persons may be infected by $>1$ isolate of Mycobacterium tuberculosis at the same time (9-11); and that transmission may occur in casual social settings (12).

Molecular epidemiologic studies have also demonstrated that secondary cases among close associates of known case-patients are not always members of the same chain of transmission, i.e., that infection may have been acquired from independent sources (13). Molecular investigations of households of multiple TB patients showed that cohabitating TB patients may be infected with distinct isolates of $M$. tuberculosis (14-16). For example, in 2 suburbs of Cape Town, South Africa, which have TB notification rates of $\approx 320$ cases per 100,000 population, researchers found that less than half $(46 \%)$ of secondary TB cases within households had a TB isolate that matched an isolate from another case within the household by RFLP (16). Overall, $<1(19 \%)$ in 5 new TB cases occurring in these communities was the result of within-household transmission.

Although studies have shown that household contacts with TB are likely to have acquired infection independently in high-incidence settings, there are no published estimates of the probability that 2 household members with multidrugresistant TB (MDR TB: resistance to at least isoniazid and rifampin) share a similar genotype and are members of the same transmission chain. Molecular epidemiologic data from households with $>1$ MDR TB case can help shed 
light on the transmissibility of highly drug-resistant disease and also help guide public health policy. For example, international guidelines for the management of known contacts of MDR TB patients recommend an empirical drug regimen based either on the drug-resistance profile of an isolate from the suspected index MDR TB casepatient or on the most common drug-resistance pattern in the community while drug sensitivity tests are pending (17-19). A better understanding of the relative importance of intrahousehold or community transmission may help to inform the choice of empirical regimen.

Despite a decreasing overall incidence of TB in Peru of $\approx 3.7 \%$ per year since 1996 , the incidence of MDR TB has increased by $\approx 4.5 \%$ over the same period (20). The increasing incidence of MDR TB in densely occupied urban communities of Lima, Peru, poses obvious challenges for TB control. We report a molecular epidemiologic study within households in Lima in which $>1$ person received a diagnosis of MDR TB. We used spoligotyping and 24-loci MIRU-VNTR typing $(21,22)$ to identify households that have had $>1$ introduction of MDR TB, and we explored the association of household factors with these multiple introduction events.

\section{Materials and Methods}

\section{Study Setting, Participants, and Data}

The estimated incidence of TB in Lima, Peru, is $>130$ cases/100,000 persons; this estimate masks substantial heterogeneity in the actual distribution of TB within this large metropolitan area where poor areas often experience several-fold higher local incidence of disease than higherincome areas (23). For example, in 2000 in northern metropolitan Lima (population 3,186,199), the incidence of active TB was 232 cases/100,000 persons (24). A nationwide survey in 2006 reported that $5.3 \%$ of all new cases and $23.6 \%$ of retreatment cases were MDR TB (25). Since 1996, Partners in Health and Socios en Salud Sucursal Peru have worked with the Peru Ministry of Health to implement a program to treat patients with active MDR TB by using supervised, individualized, antimicrobial drug regimens delivered on an ambulatory basis (26-28).

We previously reported the TB incidence in a cohort of household contacts of the patients treated for MDR TB (29). A household was eligible for inclusion in the study if $\geq 2$ members had been treated for MDR TB by this program during 1996-2004, and if $\geq 1$ MDR $M$. tuberculosis isolate obtained from each person was available for analysis. All available (pretreatment and ongoing treatment) MDR isolates from patients in eligible households were included in this analysis. Demographic data, drug-susceptibility test results, and information about the physical condition of the household structure were abstracted from the electronic records of the MDR TB program. This study was reviewed and approved by the Committee on Human Studies of the Office of Research Subject Protection of Harvard Medical School.

\section{Laboratory Methods and Drug-Susceptibility Testing}

Drug-susceptibility testing and genotyping by using MIRU-VNTR and spoligotyping were performed by the Supranational Reference Laboratory at the University of Massachusetts Medical School. A standard agar plate proportion method was used for drug-susceptibility testing of $M$. tuberculosis isolates. The first-line and second-line drugs tested were isoniazid $(0.2 \mathrm{mg} / \mathrm{L}, 1.0 \mathrm{mg} / \mathrm{L}$, and $5.0 \mathrm{mg} / \mathrm{L})$, rifampin $(1.0 \mathrm{mg} / \mathrm{L})$, streptomycin $(2.0 \mathrm{mg} / \mathrm{L}$ and $10.0 \mathrm{mg} / \mathrm{L})$, ethambutol $(5.0 \mathrm{mg} / \mathrm{L})$, kanamycin $(5.0$ $\mathrm{mg} / \mathrm{L})$, ethionamide $(10.0 \mathrm{mg} / \mathrm{L})$, capreomycin $(10.0$ $\mathrm{mg} / \mathrm{L})$, ofloxacin $(2.0 \mathrm{mg} / \mathrm{L})$, and $p$-amino salicylic acid $(8.0 \mathrm{mg} / \mathrm{L})$. Susceptibility to pyrazinamide $(100 \mathrm{mg} / \mathrm{L})$ was determined by using the BACTEC 460 Liquid Medium System (Becton Dickinson, Sparks, MD, USA). We only included drugs to which resistance had been tested for $\geq 70 \%$ of isolates in the study.

\section{MIRU-VNTR Genotyping}

DNA for PCR analysis was prepared by using a simple thermolysis procedure. PCR amplification of the 24 MIRU-VNTR loci was conducted as described $(22,30)$ with minor modifications. The PCR mixture contained 2 $\mu \mathrm{L}$ of thermolysate, $1 \times$ PCR buffer, $1 \mathrm{~mol} / \mathrm{L}$ betaine, 0.5 U Taq DNA polymerase (Takara Bio, Madison, WI, USA), $200 \mu \mathrm{mol} / \mathrm{L}$ of each $\mathrm{dNTP}$, and $0.3 \mu \mathrm{mol} / \mathrm{L}$ of each flanking primer.

An ABI Thermal Cycler 2720 (Applied Biosystems, Foster City, CA, USA) was used for PCRs. Initial denaturation at $94^{\circ} \mathrm{C}$ for 5 min was followed by 35 cycles of denaturation at $94^{\circ} \mathrm{C}$ for $30 \mathrm{~s}$, annealing at $62^{\circ} \mathrm{C}$ for $30 \mathrm{~s}$, and elongation at $70^{\circ} \mathrm{C}$ for $45 \mathrm{~s}$; and a final extension step at $72^{\circ} \mathrm{C}$ for $10 \mathrm{~min}$. M. tuberculosis H37RV DNA and sterile distilled water were included in each test run as positive and negative controls, respectively.

PCR products were analyzed in 2 ways. First, DNA fragments from amplification with primers specific for loci ETRA, ETRB, ETRC, ETRD, MIRU2, MIRU20, MIRU23, MIRU24, MIRU26, Mtub21, Mtub29, Mtub30, Mtub34, and Qub11b were separated by using standard 2\% agarose gel electrophoresis. Second, DNA fragments from amplification with primers specific for loci ETRE, MIRU10, MIRU16, MIRU27, MIRU39, MIRU40, Mtub04, Mtub39, Qub26, and Qub4156 were analyzed by electrophoresis with the QIAxcel System and the QIAxcel DNA Screening Kit (both from QIAGEN, Valencia, CA, USA). 


\section{Spoligotyping}

Mycobacterial DNA was prepared by using the same thermolysis protocol as for MIRU-VNTR typing. For DNA amplification, $0.15 \mu \mathrm{L}$ Tth polymerase $(5 \mathrm{U} / \mu \mathrm{L}$; Roche, Pleasanton, CA, USA) was added to $50 \mu \mathrm{L}$ of PCR mixture, and the following amplification profile was used: $3 \mathrm{~min}$ at $96^{\circ} \mathrm{C} ; 35$ cycles for $1 \mathrm{~min}$ at $96^{\circ} \mathrm{C}, 1 \mathrm{~min}$ at $55^{\circ} \mathrm{C}$, and $30 \mathrm{~s}$ at $72^{\circ} \mathrm{C}$; and $5 \mathrm{~min}$ at $72^{\circ} \mathrm{C}$.

Spacer oligonucleotide typing was performed by using the Multianalyte Profiling System (Luminex Inc., Austin, TX, USA). The procedure was conducted according to the protocol reported by Cowan et al. (31) with adaptations for a 96-well format. Fluorescence signals indicating hybridization strength were analyzed by using Bio-Plex Suspension Array System Instrument Luminex 100xMAP Technology (Luminex Molecular Diagnostics Inc., Toronto, Ontario, Canada) and the Bio-Rad BioPlex Manager Program version 4.1.1 (Bio-Rad Laboratories, Hercules, CA, USA). Lineage and the shared type for each isolate were assigned based on matching the spoligotype patterns with those listed in the SpolDB4 database (32).

\section{Identification of Multiple Introductions of M. tuberculosis into a Household}

Households were classified as having evidence of repeated introduction of TB from the community if isolates from $\geq 2$ patients with MDR TB within 1 household had different molecular genotypes. Supply et al. proposed a standard approach for characterizing the relatedness of $M$. tuberculosis isolates by spoligotyping and 24-loci MIRUVNTR. They found that the combination of these methods (which requires including $\geq 15$ of the most diverse loci for MIRU-VNTR analysis) has comparable discriminatory power to IS6110 RFLP typing (22). We present minimum and maximum estimates of the proportion of households judged to have evidence of multiple TB introductions on the basis of spoligotyping and MIRU-VNTR genotyping data.

We also examined a classification approach recently used by Narayanan et al. (7). Nonmatching strains are defined as those strains with $>1$ spoligotype spacer or $>1$ MIRU-VNTR locus difference. Enabling different degrees of stringency in calling 2 (or more) strains a match reflects our underlying uncertainty about how rapidly spoligotypes and MIRU-VNTR genotypes change because of mutations at marker loci during the natural history of disease and through chains of transmission that may span decades.

\section{Identification of Reinfection Events}

We genotyped all available MDR isolates of patients within study households. Among participants from whom $\geq 2$ isolates were available, we identified episodes of reinfection on the basis of differences in genotypes.
We used a similar approach for comparing genotypes for identifying episodes of reinfection and repeated household introduction.

\section{Statistical Analysis}

SAS version 9.2 (SAS, Cary, NC, USA) was used for statistical analysis. We performed standard nonparametric tests for assessing univariate associations between household-level factors and the probability of repeated introduction.

\section{Results}

We identified 105 households in which $\geq 1$ MDR $M$. tuberculosis isolate was available from each of $\geq 2$ different household members. In total, 391 MDR isolates from 236 persons were available for molecular typing. Spoligotyping and MIRU-VNTR analyses were successfully completed on samples from $\geq 2$ participants from 101 (96\%) of these households. These analyses resulted in a set of $384(98 \%)$ isolates from 232 (98\%) persons. Characteristics of persons and households included in the study are shown in Table 1. There were an additional 142 households for which we knew of $\geq 2$ patients with MDR TB, but for whom M. tuberculosis specimens were no longer available for genetic analysis. No statistically significant differences in size, density, or age distribution of members were found between the households that were included and those not included in this study.

Of 384 isolates, 228 (59\%) were tested for susceptibility to a sufficient number of second-line drugs to identify extensively drug-resistant $M$. tuberculosis strains (MDR plus additional resistance to a fluoroquinolone and a second-line, injectable antimicrobial drug [either kanamycin, amikacin, or capreomycin]). Thirty-one (14\%) of these 228 isolates were confirmed as extensively drug resistant and were obtained from 15 patients, none of whom were living in the same household.

\section{Multiple Introductions of MDR M. tuberculosis into Households}

Using a permissive definition of matching in which we included strains that differed by 1 spoligotype spacer to be matched, we estimated that $10(10 \%)$ of households had

\begin{tabular}{lc}
\hline Table 1. Characteristics of 101 households with MDR TB, Lima, \\
Peru, 1996-2004* \\
\hline Characteristic & Median (IQR) \\
\hline Persons per household & $8(6-10)$ \\
Persons per bedroom & $2.5(1.75-4.33)$ \\
Participants per household & $2(2-2)$ \\
\hline Participants, $\mathrm{n}=232$ & $23.8(19.2-30.5)$ \\
Age, y & 57.2 \\
\multicolumn{2}{l}{ Male sex, \% }
\end{tabular}


distinct MDR isolates and showed evidence of repeated introduction. The strictest definition of matching, which required exact matches in spoligotype and at all 24-loci of the MIRU-VNTR analysis, showed that 38 (38\%) of households had evidence of repeat introduction of MDR TB from the community (Figure). Using the approach of Narayanan et al. (7) for identifying nonmatching strains (pairs with $>1$ spoligotype spacer or 1 MIRU-VNTR locus difference), we classified $16(16 \%)$ households as settings with multiple introductions of MDR TB.

The 16 households in which $\geq 2$ persons had an MDR M. tuberculosis isolate that was different from that obtained from another person in the household, according to the definition of Narayanan et al. (7), are shown in online Appendix Table 1 (www.cdc.gov/EID/content/17/6/969appT1.htm). Seven of these households also had evidence of within-household transmission of MDR TB. Closer inspection of spoligotypes isolated from these households indicated that 6 of the 16 households, although failing to meet the proposed criterion for matching, had similar isolates (households 112, 192, 557, 960, 263, and 645). If these 6 households are classified as having evidence of within-household transmission, our best estimate of the number of households with evidence of multiple introductions of MDR strains is reduced to $10(10 \%)$. Under these criteria, the percentage of households with only evidence of probable within-household transmission is $90 \%$.

We used the 10 households as our most conservative set of households with evidence of multiple introductions of MDR stains and searched for household factors that were associated with multiple introduction events. We did not find any significant associations; specifically, the size and density of households, the quality of the household structure, and time span over which isolates were accrued from households all appeared to be unrelated to multiple introductions (Table 2). In addition, no significant difference was found in the number of drugs to which the isolate from the first patient was resistant between households that had repeated introduction (mean 5.1 drugs) and households that had evidence of probable within-household transmission (mean 5.3 drugs; $p=0.75$ ).

\section{Evidence of MDR Reinfection}

Ninety persons had $>1$ MDR TB isolate available for analysis. Using the definition of matching strains of Narayanan et al. (7), we found that $5(6 \%)$ of these persons had 2 distinct strains of MDR M. tuberculosis during the period of follow-up and the remaining 85 (94\%) showed repeated isolation of the same MDR strain (online Appendix Table 2, www.cdc.gov/EID/content/17/6/969appT2.htm). Closer inspection of the isolates available from these 5 persons showed that 1 person (a 20 -year-old man)

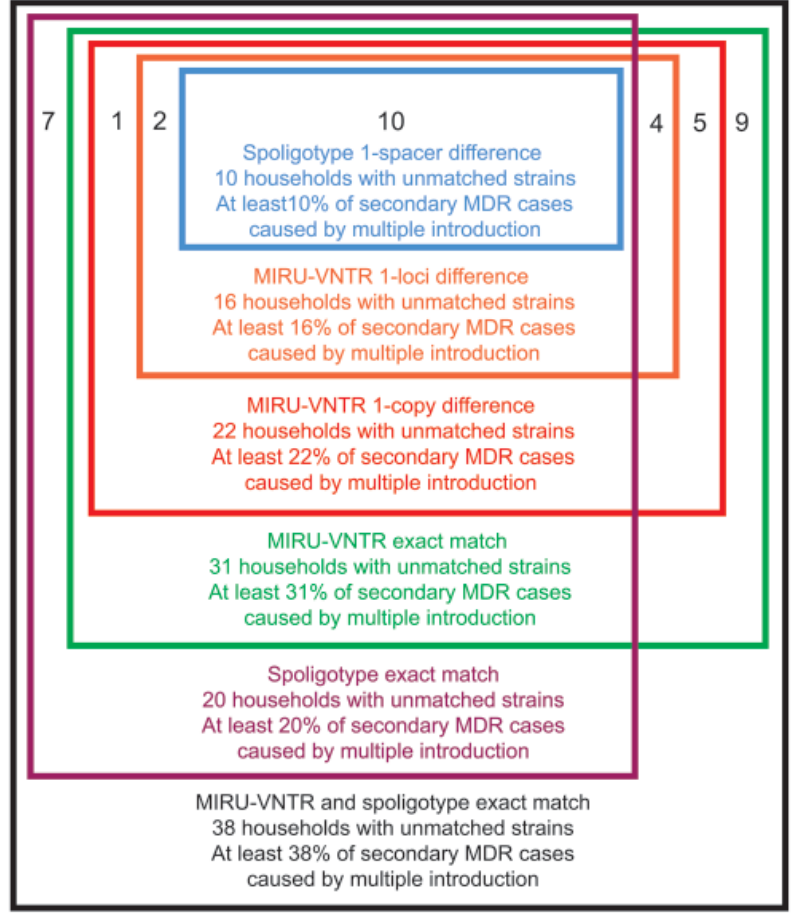

Figure. Numbers of households classified as having multiple multidrug-resistant (MDR) tuberculosis introductions by 6 definitions of matching genotypes, Lima, Peru, 1996-2004. MIRUVNTR, mycobacterial interspersed repetitive unit-variable number tandem repeat.

from household 977 may not have been reinfected. Three isolates were available from this person. The first isolate had a slightly different spoligotype than the 2 isolates subsequently obtained, but the MIRU-VNTR pattern was the same for all 3 isolates.

\section{Discussion}

In the absence of molecular epidemiologic data, secondary cases of MDR TB within a household are generally assumed to be the result of within-household transmission. In an area with increasing incidence of MDR TB (20), we found that $90 \%$ of household contacts of MDR TB index cases with active disease and drug-susceptibility test results had MDR TB (29). Our present study, in a subset of that cohort, used genotyping on the basis of spoligotyping and 24-loci MIRU-VNTR, which has been shown in other settings to have comparable discriminatory power to IS6110 RFLP (21). Our study shows that there was at least a $10 \%$ risk that a subsequent case of MDR TB occurring within the home of a known MDR TB patient was the result of transmission in the community rather than transmission in the household. This estimate represents a lower boundary of the contribution of community transmission to the appearance of secondary MDR cases 
Table 2. Association between household factors and repeated introduction of MDR TB, Lima, Peru, 1996-2004*

\begin{tabular}{|c|c|c|c|}
\hline Factor & Introduction, $\mathrm{n}=10 \dagger$ & No introduction, $\mathrm{n}=91$ & $\mathrm{p}$ value \\
\hline No. persons & $7.5(6-8)$ & $8(7-11)$ & 0.18 \\
\hline Persons per bedroom & $2.6(1.7-2.7)$ & $2.4(1.75-5)$ & 0.43 \\
\hline Homes of substandard quality $\ddagger$ & $1 / 9(11)$ & 23/64 (36) & 0.44 \\
\hline Mean age of household members, y & $28(23-32)$ & $26(21-30)$ & 0.39 \\
\hline Duration between first and last isolate obtained from household, $d$ & $389(167-724)$ & $345(204-599)$ & 0.92 \\
\hline
\end{tabular}

within a home because matching strains within a household (which we would categorize as within-home transmission) may be caused by transmission from other sources in the community. Because circulating MDR strains were heterogeneous (Table 3 ), the magnitude of this bias may not be substantial.

We did not find any easily measured household factors associated with risk for repeated introductions compared with within-home transmission. We had hypothesized that a high household density (persons/bedroom) or low quality of household structure may be associated with a higher probability of within-home transmission, conditional upon observing multiple cases within a home, but this hypothesis was not supported by these data. This finding may reflect an absence of this association between household characteristics and risk for within-home transmission or, alternatively, it may reflect the relatively small number of repeated introduction events that we observed and our limited power to test such associations. Accordingly, although our observations provide convincing evidence that repeated introduction of MDR TB into households occurs in these settings, further studies are needed to determine whether household factors, number of persons within these households, or strains present within these households are associated with an increased risk for withinhome transmission or repeated exposure in the community.

Genetic (33) or acquired susceptibility (34) to infection and disease may play a role in the accumulation of multiple TB cases within households. Because household members are likely to share genetic or environmental risk factors, or both, persons living with TB case-patients may be particularly likely to be infected and acquire disease whether they are infected by their household contact or in the community.

Our findings provide evidence to support international guidelines for management of active TB among contacts of known MDR TB cases (17-19) because they confirm that among strains from persons for which genotyping test results are available, $\leq 90 \%$ of household contacts with MDR TB were infected with the same strain as the index patient. Our findings also highlight limitations associated with such policies. Because subsequent cases of MDR TB in a household may be caused by community transmission, policies that specify that apparent secondary case-patients receive therapy on the basis of the drug-susceptibility profile of an isolate from the initial MDR TB patient may result either in effective drugs being needlessly withheld or in administration of drugs to which the strain is already resistant. This policy may result in acquisition of additional resistance to second-line drugs and prolonged opportunity for transmission of highly drug-resistant strains within homes and in the community $(35,36)$.

These findings support the use of rapid drugresistance tests to determine drug susceptibility profiles in known contacts of MDR TB patients. Molecular tests for resistance, such as line probe assays and cartridgebased PCRs (i.e., GeneXpert; Cepheid, Sunnyvale, CA, USA), are promising and have been endorsed by the World Health Organization for determining resistance to first-line drugs (37). However, although new diagnostic tests in development also detect resistance to second-line drugs $(38,39)$, these tests have not yet been optimized for use in guiding clinical care. New rapid phenotypic tests for resistance, such as the microscopic-observation drugsusceptibility assay, have also not yet been adequately tested under field conditions for their capacity to be used in selection of tailored regimens for MDR TB (40). Known contacts of MDR TB patients should be a high-priority,

\begin{tabular}{lc}
\hline $\begin{array}{l}\text { Table 3. Strain lineages of Mycobacterium tuberculosis detected } \\
\text { in the study population, Lima, Peru, 1996-2004 }\end{array}$ \\
\hline Lineage & No. (\%) \\
\hline Beijing & $19(4.9)$ \\
H1 & $22(5.7)$ \\
H3 & $22(5.7)$ \\
LAM1 & $25(6.5)$ \\
LAM3 & $12(3.1)$ \\
LAM4 & $6(1.6)$ \\
LAM5 & $47(12.2)$ \\
LAM9 & $38(9.9)$ \\
T1 & $85(22.1)$ \\
T2 & $19(4.9)$ \\
T5_MAD2 & $2(0.5)$ \\
U & $1(0.3)$ \\
X3 & $17(4.4)$ \\
\hline No match & $69(18.0)$ \\
\hline
\end{tabular}


high-yield study population for assessing the immediate utility of these new tools.

A limitation of our study is that we cannot definitively distinguish the 2 mechanisms by which distinct MDR isolates may appear within households. First, household members may have been infected by different drugsusceptible strains in the community and acquired drug resistance through deficient drug treatment. Second, household members may have been directly infected by different MDR strains in the community. Distinguishing between these 2 possibilities is essential because each would cause a distinct public health response. The first mechanism suggests that detailed investigation of individual-level or household-level risk factors for acquisition of MDR TB was needed and would indicate a need for greater treatment support and supervision for patients with drug-susceptible disease. The second mechanism indicates a need to improve infection control in the community or to facilitate diagnosis and effective treatment for persons with MDR TB to reduce the duration of infectiousness. In most circumstances, we expect acquisition and transmission to contribute to the appearance of multiple cases of MDR TB within homes, and efforts to reduce the incidence of drug-resistant disease will need to address these factors.

Although we have insufficient data for previous TB episodes and treatment for persons in our study to exclude possible independent acquisition of MDR TB among household members because of inadequate treatment, our finding that $\geq 4$ persons showed evidence of reinfection by a second (i.e., different) MDR TB strain provides evidence that there is a high risk for MDR TB exposure in this community. HIV status was known for only $\approx 50 \%$ of the persons in the study. Among those tested, only $3(3 \%)$ of 102 were HIV infected and none of the $3 \mathrm{HIV}$-infected persons were among persons in households in which multiple introductions of MDR TB were detected. If coinfection with HIV was common, it would be expected to increase the probability of rapid progression to disease and lead to higher risks of multiple cases of unlinked disease within households. Because HIV co-infection was so rare, it is unlikely that this explains the study results.

Our results extend findings from previous studies showing that a substantial fraction of cohabiting persons have independently acquired $\mathrm{TB}$ in the community (13-16). In contrast to earlier studies that compared relative contributions of within-home and community transmission, all persons in our study had MDR TB. We found that although $90 \%$ of households had evidence of intrahousehold transmission, $10 \%$ had $\geq 2$ independent introductions of MDR $M$. tuberculosis strains from the community. This finding suggests that the risk for community or extrahousehold transmission of MDR TB in Lima is high. Furthermore, it indicates that known MDR
TB contacts initiating empirical treatment for MDR TB treatment require access to drug susceptibility testing to ensure that they receive the drugs to which their isolate is susceptible. National TB programs should be wary of applying empirical regimens on the basis of populationlevel drug susceptibility data without better understanding of the relative role of intrahousehold and community transmission of MDR TB.

This study was supported by the Charles H. Hood Foundation, the David Rockefeller Center for Latin American Studies at Harvard University, and National Institutes of Health grants K01 HL080939 and U19 A1-076217.

Dr Cohen is an assistant professor in the Division of Global Health Equity at Brigham and Women's Hospital and the Department of Epidemiology at Harvard School of Public Health. His research interests are transmission dynamics and control of drug-resistant tuberculosis.

\section{References}

1. Mathema B, Kurepina NE, Bifani PJ, Kreiswirth BN. Molecular epidemiology of tuberculosis: current insights. Clin Microbiol Rev. 2006;19:658-85. doi:10.1128/CMR.00061-05

2. Barnes PF, Cave MD. Molecular epidemiology of tuberculosis. N Engl J Med. 2003;349:1149-56. doi:10.1056/NEJMra021964

3. Murray M, Nardell E. Molecular epidemiology of tuberculosis: achievements and challenges to current knowledge. Bull World Health Organ. 2002;80:477-82.

4. Alland D, Kalkut GE, Moss AR, McAdam RA, Hahn JA, Bosworth W, et al. Transmission of tuberculosis in New York City. An analysis by DNA fingerprinting and conventional epidemiologic methods. N Engl J Med. 1994;330:1710-6. doi:10.1056/ NEJM199406163302403

5. Small PM, Hopewell PC, Singh SP, Paz A, Parsonnet J, Ruston DC, et al. The epidemiology of tuberculosis in San Francisco. A population-based study using conventional and molecular methods. N Engl J Med. 1994;330:1703-9. doi:10.1056/NEJM199406163302402

6. Glynn JR, Yates MD, Crampin AC, Ngwira BM, Mwaungulu FD, Black GF, et al. DNA fingerprint changes in tuberculosis: reinfection, evolution, or laboratory error? J Infect Dis. 2004;190:1158-66. doi:10.1086/423144

7. Narayanan S, Swaminathan S, Supply P, Shanmugam S, Narendran G, Hari L, et al. Impact of HIV infection on the recurrence of tuberculosis in south India. J Infect Dis. 2010;201:691-703. doi: $10.1086 / 650528$

8. van Rie A, Warren R, Richardson M, Victor TC, Gie RP, Enarson DA, et al. Exogenous reinfection as a cause of recurrent tuberculosis after curative treatment. N Engl J Med. 1999;341:1174-9. doi:10.1056/NEJM199910143411602

9. Braden CR, Morlock GP, Woodley CL, Johnson KR, Colombel AC, Cave MD, et al. Simultaneous infection with multiple strains of Mycobacterium tuberculosis. Clin Infect Dis. 2001;33:e42-7. doi:10.1086/322635

10. García de Viedma D, Alonso Rodriguez N, Andres S, Ruiz Serrano MJ, Bouza E. Characterization of clonal complexity in tuberculosis by mycobacterial interspersed repetitive unit-variable-number tandem repeat typing. J Clin Microbiol. 2005;43:5660-4. doi:10.1128/ JCM.43.11.5660-5664.2005 
11. Shamputa IC, Rigouts L, Eyongeta LA, El Aila NA, van Deun A, Salim AH, et al. Genotypic and phenotypic heterogeneity among Mycobacterium tuberculosis isolates from pulmonary tuberculosis patients. J Clin Microbiol. 2004;42:5528-36. doi:10.1128/ JCM.42.12.5528-5536.2004

12. Munch Z, Van Lill SW, Booysen CN, Zietsman HL, Enarson DA, Beyers N. Tuberculosis transmission patterns in a high-incidence area: a spatial analysis. Int J Tuberc Lung Dis. 2003;7:271-7.

13. Dahle UR, Nordtvedt S, Winje BA, Mannsaaker T, Heldal E, Sandven $P$, et al. Tuberculosis in contacts need not indicate disease transmission. Thorax. 2005;60:136-7. doi:10.1136/thx.2004.030841

14. Bennett DE, Onorato IM, Ellis BA, Crawford JT, Schable B, Byers $\mathrm{R}$, et al. DNA fingerprinting of Mycobacterium tuberculosis isolates from epidemiologically linked case pairs. Emerg Infect Dis. 2002;8:1224-9.

15. Borrell S, Espanol M, Orcau A, Tudo G, March F, Cayla JA, et al. Factors associated with differences between conventional contact tracing and molecular epidemiology in study of tuberculosis transmission and analysis in the city of Barcelona, Spain. J Clin Microbiol. 2009;47:198-204. doi:10.1128/JCM.00507-08

16. Verver S, Warren RM, Munch Z, Richardson M, van der Spuy GD, Borgdorff MW, et al. Proportion of tuberculosis transmission that takes place in households in a high-incidence area. Lancet. 2004;363:212-4. doi:10.1016/S0140-6736(03)15332-9

17. World Health Organization. Guidelines for the programmatic management of drug-resistant tuberculosis: emergency update 2008. WHO/HTM/TB/2008.402.Geneva: The Organization; 2008.

18. World Health Organization. Treatment of tuberculosis: guidelines. 4th ed. WHO/HTM/TB/2009.420. Geneva: The Organization; 2009.

19. Tuberculosis Coalition for Technical Assistance. International standards for tuberculosis care (ISTC). The Hague: Tuberculosis Coalition for Technical Assistance; 2006 [cited 2011 Mar 10]. http://www. who.int/tb/publications/2006/istc_report.pdf

20. Dye C. Doomsday postponed? Preventing and reversing epidemics of drug-resistant tuberculosis. Nat Rev Microbiol. 2009;7:81-7. doi:10.1038/nrmicro2048

21. Oelemann MC, Diel R, Vatin V, Haas W, Rusch-Gerdes S, Locht $\mathrm{C}$, et al. Assessment of an optimized mycobacterial interspersed repetitive-unit-variable-number tandem-repeat typing system combined with spoligotyping for population-based molecular epidemiology studies of tuberculosis. J Clin Microbiol. 2007;45:691-7. doi:10.1128/JCM.01393-06

22. Supply P, Allix C, Lesjean S, Cardoso-Oelemann M, Rusch-Gerdes S, Willery E, et al. Proposal for standardization of optimized mycobacterial interspersed repetitive unit-variable-number tandem repeat typing of Mycobacterium tuberculosis. J Clin Microbiol. 2006;44:4498-510. doi:10.1128/JCM.01392-06

23. Sanghavi DM, Gilman RH, Lescano-Guevara AG, Checkley W, Cabrera LZ, Cardenas V. Hyperendemic pulmonary tuberculosis in a Peruvian shantytown. Am J Epidemiol. 1998;148:384-9.

24. Salud del Perú M. Tuberculosis en el Perú: Informe 2000. Lima (Peru): Dirección General de Salud de las Personas; 2001.

25. World Health Organization. Anti-tuberculosis drug resistance in the world: fourth global report. The WHO/IUATLD global project on anti-tuberculosis drug resistance surveillance. WHO/HTM/ TB/2008.394. 2008 [cited 2011 Mar 28]. http://www.who.int/tb/ publications/2008/drs_report4_26feb08.pdf

26. Mitnick C, Bayona J, Palacios E, Shin S, Furin J, Alcantara F, et al. Community-based therapy for multidrug-resistant tuberculosis in Lima, Peru. N Engl J Med. 2003;348:119-28. doi:10.1056/NEJMoa022928

27. Mitnick CD, Shin SS, Seung KJ, Rich ML, Atwood SS, Furin JJ, et al. Comprehensive treatment of extensively drug-resistant tuberculosis. N Engl J Med. 2008;359:563-74. doi:10.1056/NEJMoa0800106
28. Shin S, Furin J, Bayona J, Mate K, Kim JY, Farmer P. Communitybased treatment of multidrug-resistant tuberculosis in Lima, Peru: 7 years of experience. Soc Sci Med. 2004;59:1529-39. doi:10.1016/j. socscimed.2004.01.027

29. Becerra MC, Franke MF, Chalco K, Arteaga F, Bayona J, Murray M, et al. Tuberculosis burden in households of patients with multidrugresistant tuberculosis and extensively drug-resistant tuberculosis: a retrospective cohort study. Lancet. 2011;377:147-52. doi:10.1016/ S0140-6736(10)61972-1

30. Le Flèche P, Fabre M, Denoeud F, Koeck JL, Vergnaud G. High resolution, on-line identification of strains from the Mycobacterium tuberculosis complex based on tandem repeat typing. BMC Microbiol. 2002;2:37. doi:10.1186/1471-2180-2-37

31. Cowan LS, Diem L, Brake MC, Crawford JT. Transfer of a Mycobacterium tuberculosis genotyping method, spoligotyping, from a reverse line-blot hybridization, membrane-based assay to the Luminex multianalyte profiling system. J Clin Microbiol. 2004;42:474-7. doi:10.1128/JCM.42.1.474-477.2004

32. Brudey K, Driscoll JR, Rigouts L, Prodinger WM, Gori A, Al-Hajoj SA, et al. Mycobacterium tuberculosis complex genetic diversity: mining the fourth international spoligotyping database (SpolDB4) for classification, population genetics and epidemiology. BMC Microbiol. 2006;6:23. doi:10.1186/1471-2180-6-23

33. Möller M, de Wit E, Hoal EG. Past, present and future directions in human genetic susceptibility to tuberculosis. FEMS Immunol Med Microbiol. 2010;58:3-26. doi:10.1111/j.1574-695X.2009.00600.x

34. Lönnroth K, Jaramillo E, Williams BG, Dye C, Raviglione M. Drivers of tuberculosis epidemics: the role of risk factors and social determinants. Soc Sci Med. 2009;68:2240-6. doi:10.1016/j.socscimed.2009.03.041

35. Furin JJ, Becerra MC, Shin SS, Kim JY, Bayona J, Farmer PE. Effect of administering short-course, standardized regimens in individuals infected with drug-resistant Mycobacterium tuberculosis strains. Eur J Clin Microbiol Infect Dis. 2000;19:132-6. doi:10.1007/ s100960050445

36. Han LL, Sloutsky A, Canales R, Naroditskaya V, Shin SS, Seung $\mathrm{KJ}$, et al. Acquisition of drug resistance in multidrug-resistant $\mathrm{Myco}-$ bacterium tuberculosis during directly observed empiric retreatment with standardized regimens. Int J Tuberc Lung Dis. 2005;9:818-21.

37. WHO endorses new rapid tuberculosis test [cited 2011 Feb 3]. http:// www.who.int/tb/laboratory/new rapid test/en/index.html

38. Hillemann D, Rusch-Gerdes S, Richter E. Feasibility of the genotype MTBDRsl assay for fluoroquinolone, amikacin-capreomycin, and ethambutol resistance testing of Mycobacterium tuberculosis strains and clinical specimens. J Clin Microbiol. 2009;47:1767-72. doi:10.1128/JCM.00081-09

39. Kiet VS, Lan NT, An DD, Dung NH, Hoa DV, van Vinh Chau N, et al. Evaluation of the MTBDRsl test for detection of second-linedrug resistance in Mycobacterium tuberculosis. J Clin Microbiol. 2010;48:2934-9. doi:10.1128/JCM.00201-10

40. Migliori GB, Matteelli A, Cirillo D, Pai M. Diagnosis of multidrugresistant tuberculosis and extensively drug-resistant tuberculosis: current standards and challenges. Can J Infect Dis Med Microbiol. 2008;19:169-72.

Address for correspondence: Ted Cohen, Division of Global Health Equity, Brigham and Women's Hospital, 641 Huntington Ave, Boston, MA 02115, USA; email: tcohen@hsph.harvard.edu

The opinions expressed by authors contributing to this journal do not necessarily reflect the opinions of the Centers for Disease Control and Prevention or the institutions with which the authors are affiliated. 